\title{
Integration Formulas of Tenth Order and Higher
}

\author{
R. R. Aidagulov and M. V. Shamolin \\ Moscow State University, Institute of Mechanics, \\ Leninskie Gory, Moscow, 119991 Russia
}

Received December 18, 2009, in final form, September 22, 2009

\begin{abstract}
Nowadays, due to the considerable growth of computer capacities, the development of more efficient quadrature formulas may seem unnecessary. However, if the calculation of each integrand value requires much computational time or we have to study the dependence of the integral on a large number of parameters the integrand is determined through, then it is necessary to use more efficient formulas.
\end{abstract}

DOI: $10.3103 / \mathrm{S} 0027132210040017$

Quadrature formulas of 8 th order for calculation of integrals of the form $\int_{b}^{a} f(x) d x$ are presented (and used) in reference-books and program packages. In fact, it is easy to calculate all the coefficients of quadrature formulas of Gauss type for the order 10 and higher. In particular, for "symmetric weights" we have $\int_{-a}^{a} \rho(x) f(x) d x$, where $\rho(x)$ is an even weight function; the degree of the polynomial for the calculation of weights is decreased by two times, more precisely, $P(x)=x^{\theta} f\left(x^{2}\right)$, where $P(x)$ is the polynomial of degree $m=2 k+\theta, k=[m / 2]$, for determination of the weights, $f(x)$ is a polynomial of degree $k$ (here [...] is the integral part).

In all practically interesting cases of approximate calculation of integrals, one can choose an even weight function so that this expression is a Gauss integral formula with this weight. In the case $a=\infty$ we assume that all integrals for power functions are determined. Thus, taking into account the evenness of the weight function, we get that integrals of odd functions (odd powers are such functions) are equal to zero.

Since the general theory does not depend on the evenness, at the beginning we present our arguments for an arbitrary weight function and search for the integration formula in the following form:

$$
I(f)=\int_{b}^{a} \rho(x) f(x) d x=S_{m}(f)+R_{m}(f), \quad S_{m}(f)=\sum_{j=1}^{m} a_{m j} f\left(x_{m j}\right) .
$$

The polynomial $P_{m}(x)=\prod_{j}\left(x-x_{m j}\right)$ is said to be the polynomial of nodes. We perform the choice of $2 m$ variables $a_{m 1}, \ldots, a_{m m}, x_{m 1}, \ldots, x_{m m}$ so that for the function $f(x)=x^{j}, j=0,1, \ldots, 2 m-1$, the error of the integral sum $R_{m}(f)$ vanishes. Such formulas are called Gauss quadrature formulas [1; 2, Section 2]. In this case the error order of a quadrature function is estimated by the value $M_{2 m}\left|\rho_{m}-\sum_{j} a_{j} x_{j}^{2 m}\right| /(2 m)$ !, where $M_{2 m}=\sup _{x}\left|f^{(2 m)}(x)\right|$.

This implies that the polynomials of nodes are mutually orthogonal in the integral metrics:

$$
\int_{b}^{a} \rho(x) P_{m}(x) P_{n}(x) d x=0, \quad m \neq n .
$$

If $\rho(x)$ is a nonnegative function with a positive integral, then the orthogonal polynomials with the coefficient 1 at the highest degree are uniquely determined by the orthogonalization method (the necessity of the condition $P_{0}(x) \equiv 1$ follows from the consideration of integrals of $\left.f(x)=P_{m}(x)\right)$. The theory of orthogonal polynomials implies that these polynomials have exactly $m$ roots in the studied interval and hence they are polynomials of nodes. Taking the function $f(x)$ from the condition

$$
f(x)=P_{m}(x) /\left(x-x_{m j}\right)=\prod_{i \neq j}\left(x-x_{m i}\right),
$$


we determine the weights

$$
a_{m j}=\frac{1}{f_{m}^{\prime}\left(x_{m j}\right)} \int_{b}^{a} \rho(x) \prod_{i \neq j}\left(x-x_{m i}\right) d x .
$$

In order to calculate the weights $a_{m j}$, one can obtain a polynomial formula of degree $m-1: a_{m j}=\phi_{m-1}\left(x_{m j}\right)$. From the computational viewpoint, it is convenient to express the values of nodes and weights through the moments up to the order $2 m$.

The method of determination of Gauss formulas was perfectly well described for the case $\rho(x) \equiv 1$ in $[1$; 2 , Section 2]. Here we bring the corresponding calculations to particular formulas for an arbitrary symmetric weight function $\rho(x)$.

Since in the even case all moments of odd order are equal to zero and we consider only them in the sequel, we determine the moments by the formulas

$$
2 \rho_{n}=\int_{-a}^{a} \rho(x) x^{2 n} d x, \quad n=0,1, \ldots .
$$

Note that, due to the evenness of the weight function $\rho(x)$, we obtain symmetric weights $a_{j}=a_{m+1-j}$ and antisymmetric nodes $x_{j}+x_{m+1-j}=0$. Therefore, it is better to introduce a new enumeration by shifting the old one so that the positive nodes were represented as $x_{1}, x_{2}, \ldots, x_{k}$ and the negative ones as $x_{-1}=-x_{1}, \ldots, x_{-k}=-x_{k}$, additionally assume $x_{0}=0$ in the case of odd $m$.

This gives

$$
\sum_{j} a_{j}=2 \rho_{0}, \quad y_{j}=x_{j}^{2}, \quad \sum_{j \leq k} a_{j} y_{j}^{n}=\rho_{n}, \quad n=1,2, \ldots, m-1, \quad k=[m / 2] .
$$

Using the cases $n=1,2, \ldots, k$ for odd $m$ and $n=0,1, \ldots, k-1$ for even $m$, we come to the following expression for $a_{j}$ :

$$
a_{j=1+\theta}=\sum_{i} \rho_{i-1+\theta} \frac{(-1)^{i+j} \Delta_{i j}}{y_{j}^{m-2 k} \Delta},
$$

where $\Delta=\prod_{i<j}\left(y_{j}-y_{i}\right)$ is the Vandermonde determinant, $\Delta_{i j}$ is the determinant obtained from $\Delta$ by cancellation of the $i$ th row and $j$ th column.

Substituting expressions (3) into equations (2), we have

$$
\sum_{l} \rho_{l-1+\theta} c_{l}^{j}=\rho_{j-1+\theta}, \quad c_{l}^{j}=\frac{\Delta_{l}^{j}}{\Delta}
$$

where the determinant $\Delta_{l}^{j}$ is obtained from the Vandermonde determinant by replacement of the $l$ th row, namely, the row $y_{1}^{l-1}, y_{2}^{l-1}, \ldots, y_{k}^{l-1}$, is replaced by the $j$ th row $y_{1}^{j-1}, y_{2}^{j-1}, \ldots, y_{k}^{j-1}$.

It is clear that $c_{l}^{j}=\Delta_{l}^{j} / \Delta$ for $l \leq k, j \leq k$, and so equations (4) give nothing. For $j=k+1, \ldots, 2 k$ we obtain a system of $k$ Diophantine equations for determination of the unknowns $y_{1}, \ldots, y_{k}$. It is also clear that $c_{l}^{j}$ is a symmetric polynomial of degree $j-l$ of the variables $y_{1}, \ldots, y_{k}$ and the maximal degree at the variable $y_{1}$ is equal to $j-k$, and the coefficient at the same variable is $(-1)^{k-l}$. Correspondingly, $c_{l}^{k+1}=(-1)^{k-l} \sigma_{k+1-l}, c_{1}^{j}=(-1)^{k-1} \sigma_{k} c_{k}^{j-1}$, where $\sigma_{k}$ are some numbers to be determined. For example, from these equations we may obtain the symmetric functions

$$
\sigma_{1}=y_{1}+y_{2}+\ldots+y_{k}, \quad \sigma_{2}=\sum_{i<j} y_{i} y_{j}, \quad \ldots, \quad \sigma_{k}=\prod_{j} y_{j} .
$$

Using linear combinations, from (4) we get the following linear equations with respect to $\sigma_{j}$ :

$$
\sum_{0 \leq j \leq k}(-1)^{j} \rho_{k+j-1+\theta} \sigma_{j}=0, \quad m=k+j-1+\theta=k+1, \ldots, 2 k .
$$


This gives the following equation for the determination of squares of nodes:

$$
\left|\begin{array}{cccc}
1 & y & \ldots & y^{k} \\
\rho_{1} & \rho_{2} & \ldots & \rho_{k+1} \\
\ldots & \ldots & \ldots & \ldots \\
\rho_{k} & \rho_{k+1} & \ldots & \rho_{2 k}
\end{array}\right|=0, \quad \theta=1, \quad\left|\begin{array}{cccc}
1 & y & \ldots & y^{k} \\
\rho_{0} & \rho_{1} & \ldots & \rho_{k} \\
\ldots & \ldots & \ldots & \ldots \\
\rho_{k-1} & \rho_{k} & \ldots & \rho_{2 k-1}
\end{array}\right|=0, \quad \theta=0 .
$$

Calculating the roots of this equation, from (2)-(4) we get the values of the weights $a_{1}, \ldots, a_{k}$ and $a_{0}=\rho_{0}-a_{1}-\ldots-a_{k}$. Also note that setting the weights of all $y_{i}$ equal to one and the weights of $\rho_{i}$ equal to $i$, we obtain everywhere uniform formulas, and the weights $a_{i}$ are equal to zero.

There also exists a symmetry for the change of the weight function $\rho(x)$ by $\rho(x) x^{2}$ with the increment of weights of the variables $\rho_{i}$ by one.

Consider small odd values in the general case in more detail. The case $k=0$ is reduced to the following formula: $\int \rho(x) f(x) d x \approx \rho_{0} f(x)$. For $k=1$ we come to formula (1) with the following values:

$$
y_{1}=\frac{\rho_{2}}{\rho_{1}}, \quad x_{1}=\sqrt{y_{1}}, \quad a_{1}=\frac{\rho_{1}}{y_{1}}=\frac{\rho_{1}^{2}}{\rho_{2}}, \quad a_{0}=\rho_{0}-\frac{\rho_{1}^{2}}{\rho_{2}}=\left|\begin{array}{l}
\rho_{0} \rho_{1} \\
\rho_{1} \rho_{2}
\end{array}\right| / \rho_{2} .
$$

Further, for $k=2$ we have

$$
\begin{aligned}
& \left(\rho_{2}^{2}-\rho_{1} \rho_{3}\right) y^{2}-\left(\rho_{3} \rho_{2}-\rho_{1} \rho_{4}\right) y+\left(\rho_{3}^{2}-\rho_{2} \rho_{4}\right)=0, \\
& D=\rho_{1}^{2} \rho_{4}^{2}-6 \rho_{1} \rho_{2} \rho_{3} \rho_{4}-3 \rho_{2}^{2} \rho_{3}^{2}+4 \rho_{2}^{3} \rho_{4}+4 \rho_{1} \rho_{3}^{3},
\end{aligned}
$$

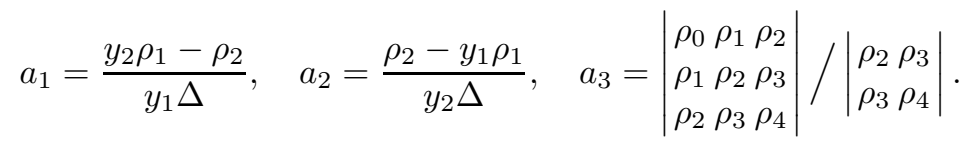

The case $k=3$ results in the following values:

$$
\begin{aligned}
& \left|\begin{array}{lll}
\rho_{1} & \rho_{2} & \rho_{3} \\
\rho_{2} & \rho_{3} & \rho_{4} \\
\rho_{3} & \rho_{4} & \rho_{5}
\end{array}\right| y^{3}-\left|\begin{array}{lll}
\rho_{1} & \rho_{2} & \rho_{4} \\
\rho_{2} & \rho_{3} & \rho_{5} \\
\rho_{3} & \rho_{4} & \rho_{6}
\end{array}\right| y^{2}+\left|\begin{array}{lll}
\rho_{1} & \rho_{3} & \rho_{4} \\
\rho_{2} & \rho_{4} & \rho_{5} \\
\rho_{3} & \rho_{5} & \rho_{6}
\end{array}\right| y-\left|\begin{array}{lll}
\rho_{2} & \rho_{3} & \rho_{4} \\
\rho_{3} & \rho_{4} & \rho_{5} \\
\rho_{4} & \rho_{5} & \rho_{6}
\end{array}\right|=0 \\
& a_{1}=\frac{\rho_{1} y_{2} y_{3}-\rho_{2}\left(y_{2}+y_{3}\right)+\rho_{3}}{y_{2}\left(y_{2}-y_{1}\right)\left(y_{3}-y_{1}\right)}, \quad a_{2}=\frac{-\rho_{1} y_{1} y_{3}+\rho_{2}\left(y_{1}+y_{3}\right)-\rho_{3}}{y_{2}\left(y_{2}-y_{1}\right)\left(y_{3}-y_{2}\right)}, \quad a_{3}=\frac{\rho_{1} y_{1} y_{2}-\rho_{2}\left(y_{1}+y_{2}\right)+\rho_{3}}{y_{3}\left(y_{3}-y_{1}\right)\left(y_{3}-y_{2}\right)} \\
& a_{0}=\rho_{0}-\frac{\rho_{1} \sigma_{2}-\rho_{2} \sigma_{1}+\rho_{3}}{\sigma_{3}}=\left|\begin{array}{cccc}
\rho_{0} & \rho_{1} & \rho_{2} & \rho_{3} \\
\rho_{1} & \rho_{2} & \rho_{3} & \rho_{4} \\
\rho_{2} & \rho_{3} & \rho_{4} & \rho_{5} \\
\rho_{3} & \rho_{4} & \rho_{5} & \rho_{6}
\end{array}\right| /\left|\begin{array}{lll}
\rho_{2} & \rho_{3} & \rho_{4} \\
\rho_{3} & \rho_{4} & \rho_{5} \\
\rho_{4} & \rho_{5} & \rho_{6}
\end{array}\right| .
\end{aligned}
$$

Now consider particular weight functions and calculate the formulas of the 10th and 14th orders of accuracy. For the 14th order of accuracy the weights and nodes are roots of cubic equations and can be expressed by Cardano formulas. Since those expressions are bulky, we present here only approximate values.

1) In some applications we have to calculate the integrals

$$
\int_{-\infty}^{\infty} f(x) \exp \left(-x^{2} / 2\right) d x
$$

In this case it is convenient to take $\rho(x)=\exp \left(-x^{2} / 2\right), a=\infty$. The corresponding orthogonal polynomials (normalized polynomials of nodes) are called Sonin polynomials, and the polynomials for the weight function $\rho(x)=\exp \left(-x^{2}\right)$ connected to the previous ones by the contraction of the axis $x$ by $\sqrt{2}$ times are called Hermitian or Chebyshev-Hermitian [3, Ch. 1; 4, Ch. 4].

The equality $\rho_{n}=\sqrt{\pi / 2}(2 n-1)$ !! is valid.

For $m=5(k=2)$ we obtain the nodes and weights for the 10th order of accuracy:

$$
x_{1}=\sqrt{5-\sqrt{10}}=-x_{-1}, \quad a_{1}=a_{-1}=\frac{7+2 \sqrt{10}}{30} \sqrt{\frac{\pi}{2}},
$$




$$
x_{2}=\sqrt{5+\sqrt{10}}, \quad a_{2}=a_{-1}=\frac{7-2 \sqrt{10}}{30} \sqrt{\frac{\pi}{2}}, \quad a_{0}=\frac{8}{15} \sqrt{\frac{\pi}{2}},
$$

and for $m=7(k=3)$ the corresponding polynomial has the form $y^{3}-21 y^{2}+105 y-105=0$, and in this case the nodes and weights are

$$
x_{1}=1.154405394739968, \quad x_{2}=2.366759410734541, \quad x_{3}=3.750439717725742,
$$

$a_{0}=0.5898718274019, \quad a_{1}=0.601899548885595, a_{2}=0.05124934362178949, \quad a_{3}=0.01029341740621618$.

2) The following integrals of hypergeometric form often occur in practice $[4$, Ch. 4$]$

$$
\begin{gathered}
\operatorname{Re} \int_{-\infty}^{\infty} \sqrt{\frac{\left(x-a_{1}\right)\left(a_{2}-x\right) \ldots\left(a_{n}-x\right)}{\left(b_{1}-x\right)\left(b_{2}-x\right) \ldots\left(b_{n}-x\right)}} e^{-c x} d x=\sum_{i} \int_{a_{i}}^{b^{i}} \frac{f_{i}(x) d x}{\sqrt{\left(x-a_{i}\right)\left(b_{i}-x\right)}}, \\
f_{i}(x)=\left(x-a_{i}\right) e^{-c x} \sqrt{\frac{\left(b_{i}-x\right)}{\left(x-a_{i}\right)}\left(\prod_{j} \frac{a_{j}-x}{b_{j}-x}\right)}, \quad a_{1}<b_{1}<a_{2}<b_{2}<\ldots<a_{n}<b_{n} .
\end{gathered}
$$

In order to calculate such integrals efficiently, one should use formulas for the weight $\frac{1}{\sqrt{1-x^{2}}}, a=1$. Then

$$
\rho_{n}=\frac{\pi}{2} \frac{(2 n-1) ! !}{(2 n) ! !}\left(\rho_{0}=\frac{\pi}{2}\right) .
$$

This case is the simplest from the computational viewpoint because the quadrature formula takes the simple form

$$
\int_{-1}^{1} \frac{f(x) d x}{\sqrt{1-x^{2}}}=\frac{\pi}{m} \sum_{j} f\left(x_{j}\right), \quad x_{l}=-\cos \frac{(2 l-1) \pi}{2 m} .
$$

The consideration of this case is reduced to the following equations

$$
\begin{gathered}
I(f)=\int_{-1}^{1} \frac{f(x) d x}{\sqrt{1-x^{2}}}=\frac{\pi}{2} \int_{-1}^{1} \sin ^{2 k}\left(\frac{\pi x}{2}\right) d x=\frac{\pi}{2} \int_{0}^{2} \cos ^{2 k}\left(\frac{\pi x}{2}\right) d x=\frac{\pi}{2^{2 k+1}} \sum_{0 \leq j \leq k} C_{2 k}^{j} \int_{0}^{2} \cos \frac{2(k-j) \pi x}{2} d x, \\
S(f)=\frac{\pi}{m} \sum_{0 \leq l \leq m} \cos ^{2 k}\left(\frac{\pi(2 l-1)}{2 m}\right)=\frac{\pi}{2^{2 k}} \sum_{0 \leq j \leq k} C_{2 k}^{j} \frac{1}{m} \sum_{0 \leq l \leq m} \cos \frac{(k-j)(2 l-1) \pi}{m} .
\end{gathered}
$$

Therefore, it remains to prove that

$$
\frac{1}{2} \int_{0}^{2} \cos (n \pi x) d x=\frac{1}{m} \sum_{0 \leq l \leq m} \cos \frac{\pi n(2 l-1)}{2 m}, \quad n<m .
$$

The validity of equality (6) follows from the consideration of the following cases: 1 ) for $n=0$ in the left- and right-hand sides of equality (6) we have $1 ; 2)$ for $0<n<m$ in the left- and right-hand sides of equality (6) we get 0 . The latter takes place due to the auxiliary multiplication of both the sides by $\cos \frac{\pi n}{2 m} \neq 0$. Actually, it is not difficult to calculate the difference between the integral and the approximative sum for $k \geq m$ :

$$
I\left(x^{2 k}\right)-S\left(x^{2 k}\right)=\frac{\pi}{2^{2 k}} \sum_{1 \leq l \leq k / m} C_{2 k}^{k-l m} .
$$

In this case (the same) weights and nodes are represented by simple formulas (5) and we have no need to consider separate cases. These normalized polynomials of nodes are called Chebyshev polynomials of the first kind.

3) $\rho(x) \equiv 1, a=1$. In this case $\rho_{n}=1 /(2 n+1)$ and the polynomials of nodes (which are Legendre polynomials up to normalization) have the form

$$
\frac{n !}{(2 n) !} \frac{d^{n}\left(x^{2}-1\right)^{n}}{d x^{n}}
$$


Correspondingly, for $k=2$ we get

$$
x_{1}=\sqrt{\frac{35-\sqrt{280}}{63}}, \quad x_{2}=\sqrt{\frac{35+\sqrt{280}}{63}}, \quad a_{1}=\frac{322+13 \sqrt{70}}{900}, \quad a_{2}=\frac{322-13 \sqrt{70}}{900}, \quad a_{0}=\frac{128}{225}
$$

for $k=3$ the equation for nodes has the form $35-315 y+639 y^{2}-429 y^{3}=0$ and the nodes and weights are

$$
\begin{gathered}
x_{1}=0.4058451513773965, \quad x_{2}=0.741531185599394, \quad x_{3}=0.949107912342758, \\
a_{0}=0.0794161895996775, \quad a_{1}=0.3818300505051187, \\
a_{2}=0.133350981823048378, \quad a_{3}=0.405402778072155431 .
\end{gathered}
$$

For calculation of the integral $\int_{-1}^{1} \cos \left(\frac{\pi}{2} x\right) d x=\frac{4}{\pi}$ these formulas give the following errors:

$$
R_{0} \approx 0.72676, \quad R_{1} \approx 8.842 \cdot 10^{-4}, \quad R_{2} \approx 7.021 \cdot 10^{-8}, \quad R_{3}<10^{-12}
$$

In principle. the latter estimates allow us to use the integration formulas of the 10th and 14th orders on a computer (and also, as before, on programmable calculators) for the latter two cases.

\section{ACKNOWLEDGMENTS}

The work was supported by the Russian Foundation for Basic Research (project no. 08-01-00231-a).

\section{REFERENCES}

1. S. M. Nikolskii, Quadrature Formulas (Fizmatgiz, Moscow, 1958) [in Russian].

2. A. N. Krylov, Lectures on Approximate Calculations (Moscow, 1950) [in Russian].

3. G. Szego, Orthogonal Polynomials (AMS, Providence, RI, 1975; Mir, Moscow 1962).

4. A. F. Nikiforov and V. B. Uvarov, Special Functions of Mathematical Physics (Nauka, Moscow, 1976) [in Russian].

Translated by V. Valedinskii 